\title{
Modified Newtonian Dynamics: A Falsification of Cold Dark Matter
}

\author{
R. H. Sanders \\ Kapteyn Astronomical Institute, University of Groningen, 9700 AV Groningen, The Netherlands \\ Correspondence should be addressed to R. H. Sanders, sanders@astro.rug.nl
}

Received 24 February 2009; Accepted 11 August 2009

Recommended by Kenneth Freeman

\begin{abstract}
The only viable alternative to dark matter is one in which Newtonian dynamics or gravity breaks down in the limit of low accelerations, as in modified Newtonian dynamics (MONDs). This hypothesis, suggested by Milgrom, has been successful in explaining systematic properties of spiral and elliptical galaxies and predicting in detail the observed rotation curves of spiral galaxies with only one additional parameter-a critical acceleration which is on the order of the cosmologically interesting value of $\mathrm{cH}_{o}$. MOND may be viewed as an algorithm for calculating the distribution of force in an astronomical object from the observed distribution of baryonic matter. The fact that it works very well on the scale of galaxies is problematic for cold dark matter (CDM). Here I present evidence in favor of this assertion and claim that this is, in effect, a falsification of CDM on the scale of galaxies.
\end{abstract}

Copyright (C) 2009 R. H. Sanders. This is an open access article distributed under the Creative Commons Attribution License, which permits unrestricted use, distribution, and reproduction in any medium, provided the original work is properly cited.

\section{Introduction}

It is evident that a significant number of physicists are willing to devote their careers to the direct detection of dark matter particles. This intense investment of time, energy, and money reflects the perceived importance of this problem and its relevance to fundamental physics. What is the identity and nature of $80 \%$ of the mass content of the Universe and what does this tell us of physics beyond the Standard Model? Yet, in spite of the monumental and creative efforts, there is no convincing evidence that dark matter particles have been detected (leaving aside the controversial DAMA result). At present, dark matter is only evidenced by its putative global gravitational effect in large astronomical systems; as long as this is true, its presumed existence is not independent of the assumed form of the law of gravity or inertia on astronomical scales. In view of this, consideration of alternatives (e.g., a theory of gravity enlarged beyond General Relativity) would not unreasonable.

The only observationally viable alternative is modified Newtonian dynamics (MONDs), an ad hoc modification of Newton's law of gravity or inertia proposed by Milgrom [1] more than 25 years ago. The phenomenological foundations for MOND come down to two observational facts about spiral galaxies. (1) The rotation curves of spiral galaxies are asymptotically flat, and (2) there is a well-defined relationship between the rotation velocity in spiral galaxies and the luminosity-the Tully-Fisher (TF) law [2]. This latter implies a mass-velocity relationship of the form $M \propto$ $V^{\alpha}$ where $\alpha$ is in the neighborhood of 4 .

The phenomenon of flat rotation curves is well known, and an example is shown in Figure 1. The rotation curve, measured in the $21 \mathrm{~cm}$ line of neutral hydrogen, extends well beyond the visible disk of the galaxy and shows no indication of a decline, certainly not a Keplerian decline.

One can conceive of several modifications which could yield flat rotation curves. But Milgrom realized that the only modification leading to a Tully-Fisher law with a logarithmic slope of four is one in which a deviation from Newton's law appears not at large distance but at low acceleration. His suggestion, viewed as a modification of gravity, was that the actual gravitational acceleration $g$ is related to the Newtonian gravitational acceleration $\mathrm{g}_{n}$ as

$$
\mathrm{g} \mu\left(\frac{|g|}{a_{o}}\right)=\mathrm{g}_{n},
$$

where $a_{o}$ is a new physical parameter with units of acceleration and $\mu(x)$ is a function which is unspecified but must 


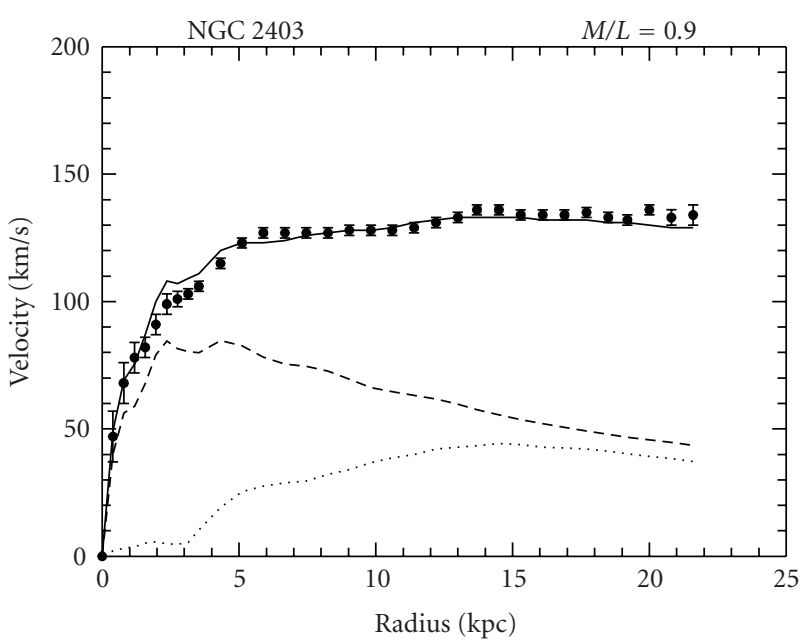

Figure 1: The $21 \mathrm{~cm}$ line rotation curve of the spiral galaxy NGC 2403 (points with error bars). The dashed curve shows the Newtonian rotation curve of the visible disk, assuming a constant mass-to-light ratio of 0.9 in solar units. The dotted curve is Newtonian rotation curve due to the gaseous component (hydrogen plus primordial helium). The solid curve is the MOND rotation curve. One should bear in mind that such theoretical curves also possess error bars, or a range of confidence, due to observational uncertainties in the baryonic mass distribution.

have the asymptotic form $\mu(x)=x$ when $x \ll 1$ and $\mu(x)=$ 1 , where $x \gg 1$.

The immediate consequence of this is that, in the limit of low accelerations, $\mathrm{g}=\sqrt{\mathrm{g}_{n} a_{0}}$. For a point mass $M$, if we set $\mathrm{g}$ equal to the centripetal acceleration $v^{2} / r$, this gives

$$
v^{4}=G M a_{o}
$$

in the low acceleration regime. So all rotation curves are asymptotically flat and there is a mass-luminosity relation of the form $M \propto v^{4}$. These are aspects that are built into MOND so they cannot rightly be called predictions. However, in the context of MOND, the aspect of an asymptotically flat rotation curve is absolute. MOND leaves rather little room for maneuver; the idea is in principle falsifiable, or at least it is far more fragile than the dark matter hypothesis. Unambiguous examples of rotation curves (of isolated galaxies) which decline in a Keplerian fashion at a large distance from the visible object would falsify the idea.

In addition, this mass-rotation velocity relation (2) forms the basis of the observed Tully-Fisher relationship, a luminosity-rotation velocity correlation of the form $L \propto$ $V^{\alpha}$. In so far as the mass-to-light ratio in spirals is roughly constant, the TF relation should be the same for different classes of galaxies and the logarithmic slope must be four. Moreover, the relation is essentially one between the total baryonic mass of a galaxy and the asymptotic flat rotational velocity-not the peak rotation velocity but the velocity at large distance. This is the most immediate and most obvious prediction $[3,4]$.

The near-infrared TF relation for the Ursa Major sample of spiral galaxies [5] is shown as a log-log plot in Figure 2,

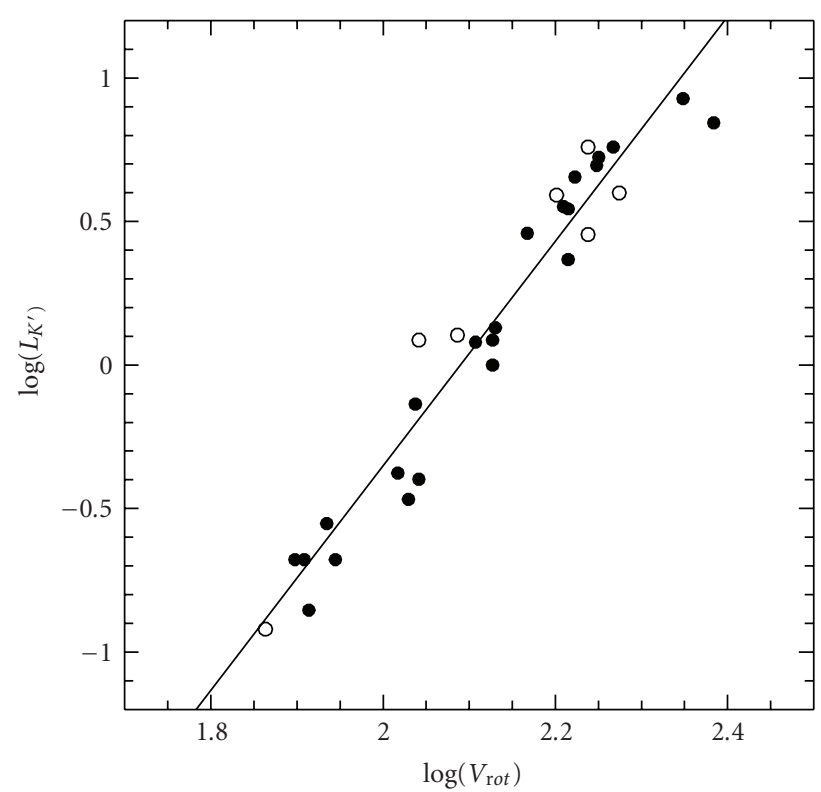

FIgURE 2: The near-infrared Tully-Fisher relation of Ursa Major spirals [5]. The rotation velocity is the asymptotically constant value. The line is a least-square fit to the data and has a slope of $3.9 \pm 0.2$.

where the velocity is that of the flat part of the rotation curve. The scatter about the least-square fit line of slope $3.9 \pm 0.2$ is consistent with observational uncertainties (i.e., no intrinsic scatter). Given the mean near-infrared mass-to-light ratio of about one, this observed TF relation (2) tells us that $a_{0}$ must be on the order of $10^{-8} \mathrm{~cm} / \mathrm{s}^{2}$. It was immediately noticed by Milgrom that $a_{o} \approx c H_{o}$ to within a factor of 5 or 6 . This cosmic coincidence suggests that MOND, if it is right, may reflect the effect of cosmology on local particle dynamics.

\section{General Predictions}

There are several other immediate consequences of modified dynamics which were previewed by Milgrom in his original papers.

(1) The critical acceleration may be rewritten as a surface density

$$
\Sigma_{m} \approx \frac{a_{o}}{G} .
$$

If a system, such as a spiral galaxy, has a surface density of matter greater than $\Sigma_{m}$, that means that the internal accelerations are greater than $a_{o}$, so the system is in the Newtonian regime. In systems with $\Sigma \geq \Sigma_{m}$ (high surface brightness or HSB galaxies) there should be a small discrepancy between the visible and classical Newtonian dynamical masses within the optical disk. But in low surface brightness (LSB) galaxies $\left(\Sigma \ll \Sigma_{m}\right)$ there is a low internal acceleration, so the discrepancy between the visible and dynamical masses would be large. By this argument Milgrom predicted, before the actual discovery of a large population of LSB galaxies, that there should be a serious discrepancy between the observable and dynamical masses within the luminous disk 
of such systems-they should exist. They do exist, and this prediction has been verified [3].

(2) Rotationally supported Newtonian systems tend to be unstable to global nonaxisymmetric modes which lead to bar formation and rapid heating of the system [6]. In the context of MOND, these systems would be those with $\Sigma>\Sigma_{m}$, so this would suggest that $\Sigma_{m}$ should appear as an upper limit on the surface density of rotationally supported systems. This critical surface density is $0.2 \mathrm{~g} / \mathrm{cm}^{2}$ or $860 M_{\odot} / \mathrm{pc}^{2}$. A more appropriate value of the mean surface density within an effective radius would be $\Sigma_{m} / 2 \pi$ or $140 M_{\odot} / \mathrm{pc}^{2}$. Taking $M / L_{b} \approx 2$, this would correspond to a surface brightness of about $22 \mathrm{mag} / \mathrm{arc} \mathrm{sec}{ }^{2}$. There is such an observed upper limit on the mean surface brightness of spiral galaxies and this is known as Freeman's law $[7,8]$. The existence of such a limit becomes understandable in the context of MOND.

(3) Spiral galaxies with a mean surface density near this limit-HSB galaxies - would be, within the optical disk, in the Newtonian regime. So one would expect that the rotation curve would decline beyond the visible disk in a near Keplerian fashion to the asymptotic constant value. In LSB galaxies, with mean surface density below $\Sigma_{m}$, the prediction is that rotation curves would rise to the final asymptotic flat value. So there should be a general difference in rotation curve shapes between LSB and HSB galaxies. In Figure 3, I show the observed rotation curves (points) of two galaxies, an LSB [9] and HSB [10], where we see exactly this trend. This general effect in observed rotation curves was first pointed out by Casertano and van Gorkom [11].

(4) With Newtonian dynamics, pressure-supported systems which are nearly isothermal have infinite extent. But in the context of MOND it is straightforward to demonstrate that such isothermal systems are finite with the density at large radii falling roughly like $1 / r^{4}$ [13]. The equation of hydrostatic equilibrium for an isotropic isothermal system reads

$$
\sigma_{r}^{2} \frac{d \rho}{d r}=-\rho \mathrm{g}
$$

where in the limit of low accelerations $\mathrm{g}=\sqrt{G M a_{0}} / r$ is the radial velocity dispersion and $\rho$ is the mass density. It then follows immediately that, in this MOND limit,

$$
\sigma_{r}^{4}=G M a_{o}\left(\frac{d \ln (\rho)}{d \ln (r)}\right)^{-2} .
$$

Thus there exists a mass-velocity dispersion relation of the form

$$
\left(M / 10^{11} M_{\odot}\right) \approx\left(\sigma_{r} / 100 \mathrm{kms}^{-1}\right)^{4},
$$

that is similar to the observed Faber-Jackson relation (luminosity-velocity dispersion relation) for elliptical galaxies [14]. This means that a MOND near-isothermal sphere with a velocity dispersion on the order of $100 \mathrm{~km} / \mathrm{s}$ will always have a galactic mass. It also means that a nearisothermal object with a velocity dispersion of $1000 \mathrm{~km} / \mathrm{s}$

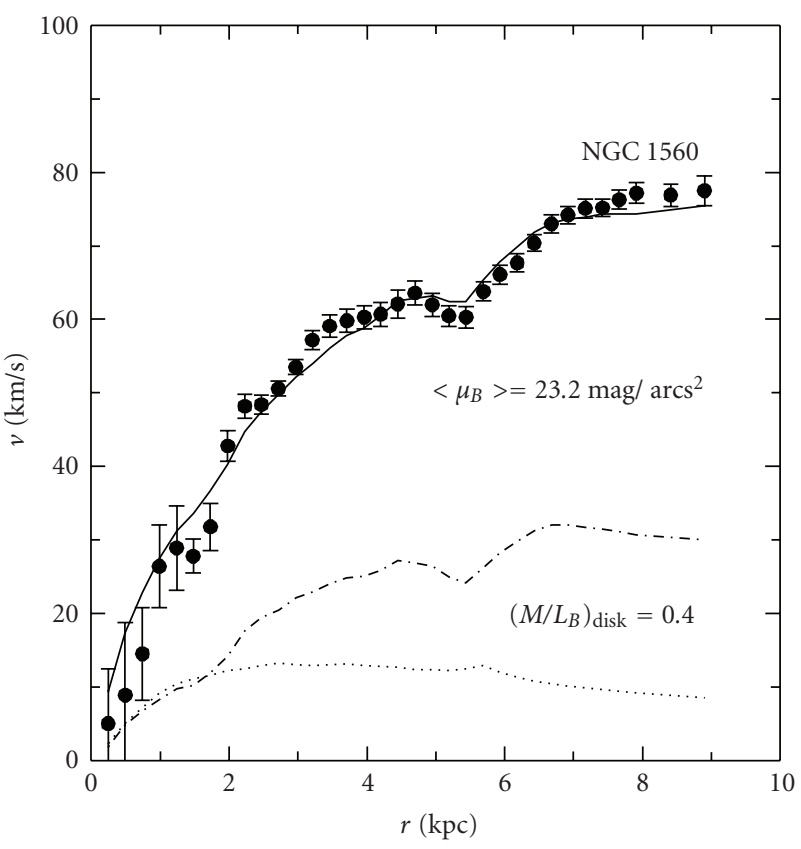

(a)

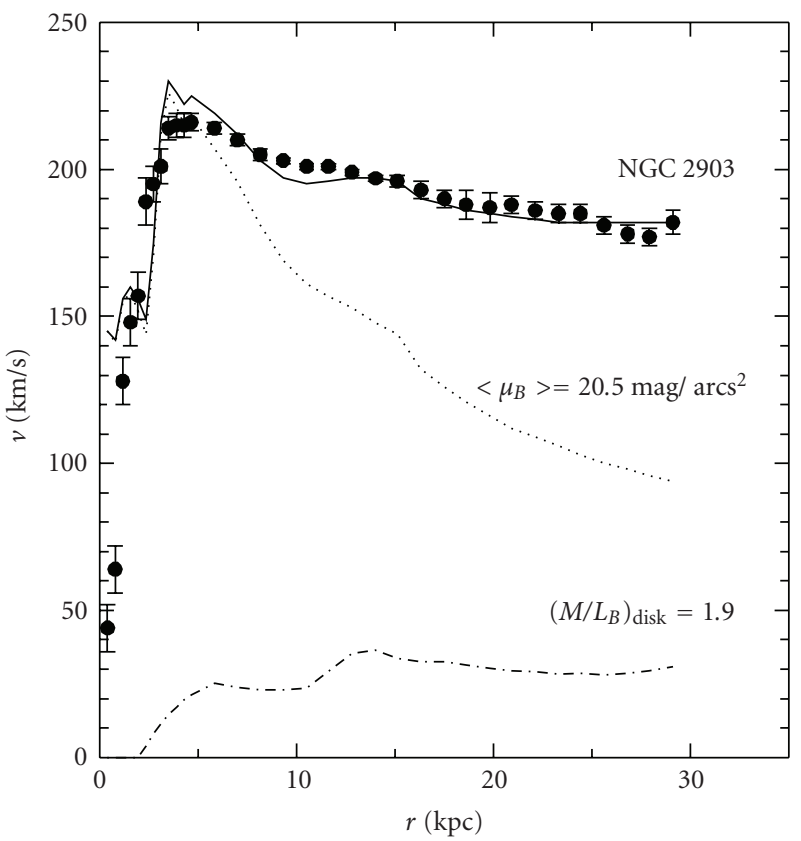

(b)

FIgURE 3: The points show the observed $21 \mathrm{~cm}$ line rotation curves of a low surface brightness galaxy, NGC 1560 [9] and a high surface brightness galaxy, NGC 2903 [10]. The dotted and dashed lines are the Newtonian rotation curves of the visible and gaseous components of the disk and the solid line is the MOND rotation curve with $a_{0}=1.2 \times 10^{-8} \mathrm{~cm} / \mathrm{s}^{2}$ - the value derived from the rotation curves of 10 nearby galaxies [12]. Here the only free parameter is the mass-to-light ratio of the visible component.

will have the mass of a cluster of galaxies, and an object with a velocity dispersion of $5 \mathrm{~km} / \mathrm{s}$ will have the mass of a globular star cluster. In other words, the same Faber-Jackson law should apply to all pressure supported near-isothermal 


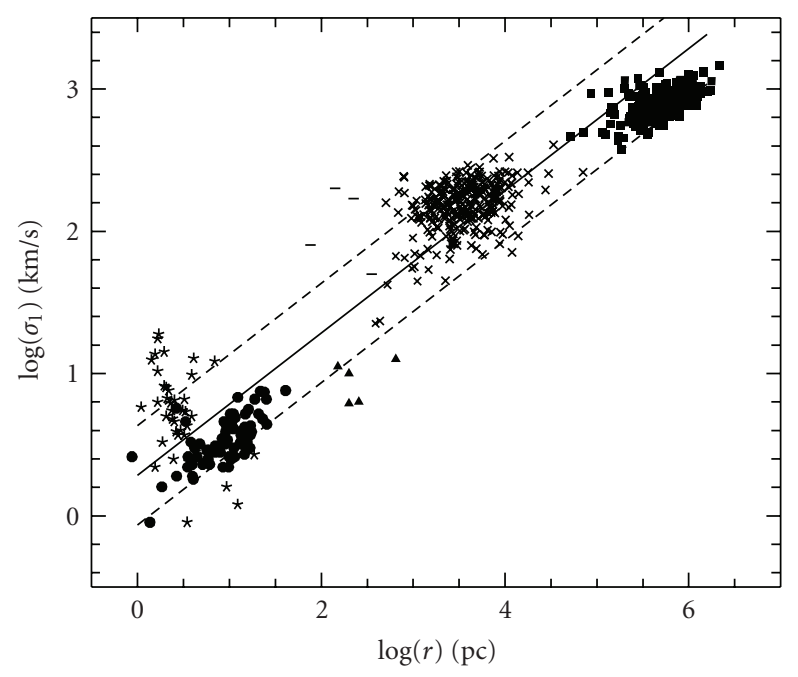

FIGURE 4: A log-log plot of the velocity dispersion in hot stellar systems against the characteristic size for different classes of objects. The star points are globular clusters, the solid round points are massive molecular clouds in the Galaxy, the crosses are luminous elliptical galaxies, the triangles are dwarf spheroidal galaxies, the dashes are compact dwarf ellipticals, and the squares are X-ray emitting clusters of galaxies. The solid line corresponds to $\sigma^{2} / r=a_{0}$ and the dashed lines show a factor of 2.2 variation on each side. The references to the relevant observational papers may be found in [15].

objects. For Newtonian systems, the existence of such a relationship must be explained by the contingencies of structure formation rather than existent dynamics. Because of the appearance of an additional dimensional constant, $a_{o}$, in the structure equation (5), MOND systems are much more constrained than their Newtonian counterparts.

But with respect to actual pressure supported systems, an even stronger statement can be made. Any isolated system that is nearly isothermal will be a MOND object. That is because a Newtonian isothermal system (with large internal accelerations) is an object of infinite size and will always extend to the region of low accelerations $\left(<a_{o}\right)$. At that point $\left(r_{e}^{2} \approx G M / a_{o}\right)$, MOND intervenes and the system will be truncated. This means that the internal acceleration of any isolated isothermal system $\left(\sigma_{r}^{2} / r_{e}\right)$ is expected to be on the order of or less than $a_{o}$ and that the mean surface density within $r_{e}$ will typically be $\Sigma_{m}$ or less (there are low-density solutions for MOND isothermal spheres, $\rho \ll a_{o}^{2} / G \sigma^{2}$, with internal accelerations less than $a_{o}$ ).

Figure 4 shows the observations [15]. This is log-log plot of velocity dispersion versus size for systems spanning many orders of magnitude from subgalactic to supergalactic systems (the identity of the systems is noted in the caption). The straight line is not a fit but rather the locus of $\sigma^{2} / r=a_{0}$. We see that the internal acceleration of these systems all lie with in a factor of a few of $a_{0}$. It is not evident how dark matter would explain this, but such a fact would certainly seem to be a challenge that should not be ignored.

\section{Rotation Curve Analysis}

The most impressive phenomenological success of MOND is in predicting the form of galaxy rotation curves from the observed distribution of detectable matter-stars and gas $[3,5,12]$. The procedure followed is described in detail by Sanders and McGaugh [15]. Basically, one assumes that light traces mass, that is, the mass-to-light ratio $(M / L)$ in any individual galaxy is constant. Then, after converting the surface brightness distribution (preferably in the near infrared) into a surface density distribution and including the contribution of the gas which is observed directly, the Newtonian gravitational force, $g_{n}$, is calculated via the classical Poisson equation. Here it is usually assumed that the stellar and gaseous disks are razor thin. The "true" gravitational force, $g_{n}$, is calculated from the MOND formula with $a_{o}$ fixed, and the mass of the stellar disk is adjusted until the best match to the observed rotation curve is achieved. This gives $M / L$ of the disk as the single free parameter of the fit (unless a bulge is present).

In comparison to the observed rotation curve one assumes that the motion of the gas is coplaner rotation about the center of the given galaxy. This is certainly not always the case because there are well-known distortions to the velocity field in spiral galaxies caused by bars and warping of the gas layer. In a fully 2-dimensional velocity field these distortions can often be modeled, but the optimal rotation curves are those in which there is no evidence for the presence of significant deviations from coplaner circular motion. But it should be remembered that a perfect theory will not fit all rotation curves because of these possible problems (the same is true of a specified dark matter halo). The point is that with MOND, usually, there is one free parameter per galaxy and that is the mass or $M / L$ of the stellar disk; with dark halos there are typically three free parameters: $M / L$ of the disk, a length scale, and velocity dispersion (or total mass) for the halo.

Figure 3 shows two examples of MOND fits to rotation curves. The dotted and dashed curves are the Newtonian rotation curves of the stellar and gaseous disks, respectively, and the solid curve is the MOND rotation curve with $a_{o}=$ $1.2 \times 10^{-8} \mathrm{~cm} / \mathrm{s}^{2}$. We see that, not only does MOND predict the general trend for LSB and HSB galaxies, but it also predicts the observed rotation curves in detail from the observed distribution of matter. This procedure has been carried out for about 100 rotation curves (see [15] for a more complete compilation of MOND rotation curves). In only about 10 cases the predicted rotation curve is significantly different from the observed curve, and for these objects there is usually an obvious problem with the observed curve or its use as a tracer of the radial force distribution. Moreover, there is the general impression that as the observational data improves, so does the agreement between the MOND and observed rotation curves (see, e.g., the higher resolution rotation curve of NGC 2903 from the THINGS survey [16]).

I have noted that the only free parameter in these fits is the mass-to-light ratio of the visible disk, so one may well ask if the inferred values are reasonable. Here it is useful to 


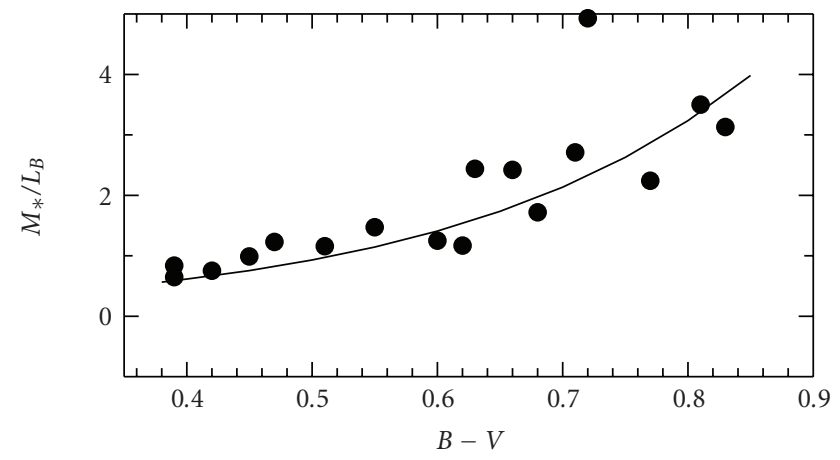

(a)

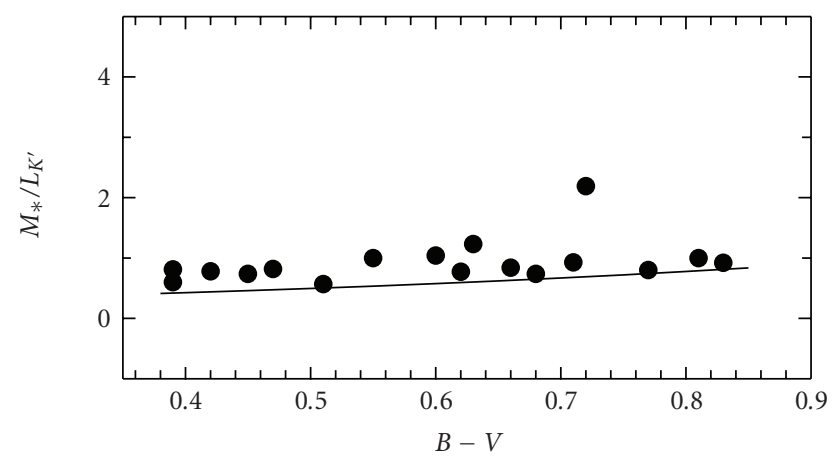

(b)

Figure 5: Inferred mass-to-light ratios for the UMa spirals in the B-band (a) and the K'-band (b) plotted against B-V colors [5]. The solid lines show predictions from populations synthesis models [16].

consider again the UMa sample [5] because all galaxies are at the same distance and there is $\mathrm{K}^{\prime}$-band (near infrared) surface photometry for the entire sample. The sample also contains both HSB and LSB galaxies. Figure 5 shows the $M / L$ in the B-band required by the MOND fits plotted against B$\mathrm{V}$ color (a) and the same for the K'-band (b). We see that in the $\mathrm{K}$ '-band $M / L \approx 1$ with a $30 \%$ scatter. In other words, if one were to assume a $\mathrm{K}^{\prime}$-band $M / L$ of one at the outset, most rotation curves would be quite accurately predicted from the observed light and gas distribution with no free parameters. In the B-band, on the other hand, the MOND $M / L$ does appear to be a function of color in the sense that redder objects have larger $M / L$ values. This is exactly what is expected from population synthesis models as is shown by the solid lines in both panels [17]. This is striking because there is nothing built into MOND which would require that redder galaxies should have a higher $M / L_{b}$; this simply follows from the rotation curve fits.

I have already shown three examples of observed rotation curves compared to the curve calculated with the MOND formula using the observed distribution of detectable baryons (Figures 1 and 3). I show one more in Figure 6 because it illustrates very well a point that I wish to emphasize. UGC 7524 is a dwarf low surface brightness galaxy [18]. In the Figure 6(a) I show the logarithm of the surface density in stars and gas as a function of radius

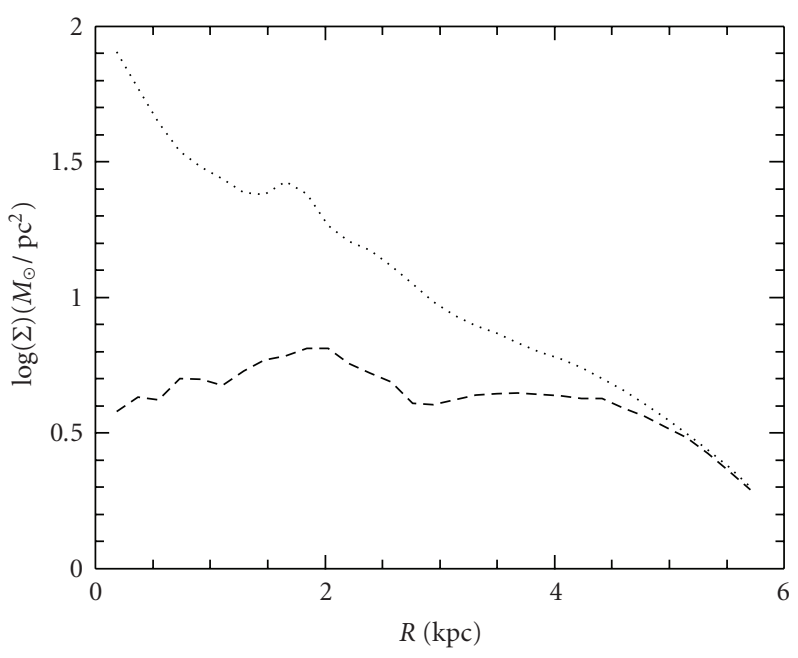

(a)

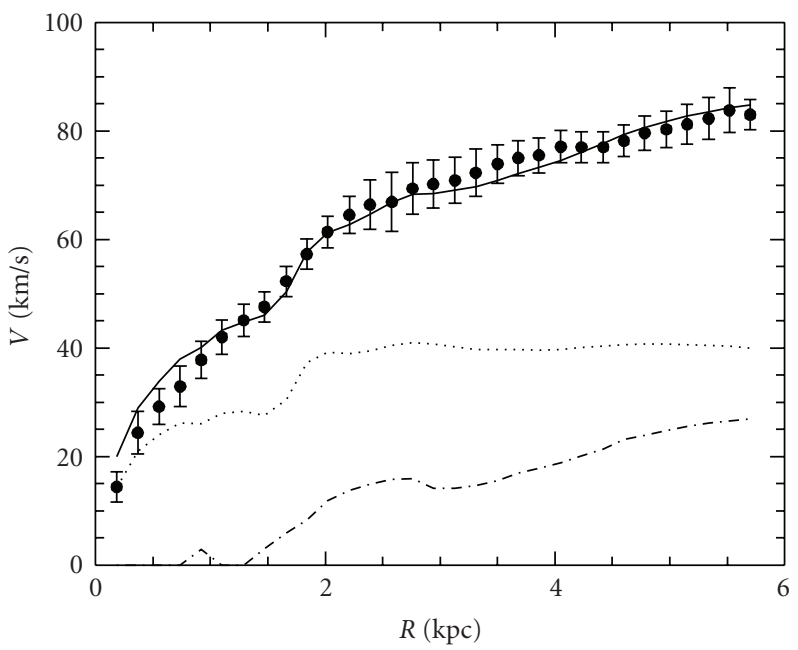

(b)

Figure 6: (a) The mass surface density distribution in stars and gas (dotted and dashed curves) as a function of radius for the low surface brightness dwarf, UGC 7524. (b) The corresponding Newtonian and the MOND rotation curves (dotted, dashed, solid). The points are the observed curve [18].

(the stellar surface density is determined from the surface brightness distribution with MOND value of $M / L=1.6$ ). In Figure 6(b) I show again the observed rotation curve (points), the Newtonian rotation curves of stars and gas, and the MOND rotation curve. We see that, for both stars and gas, there is an enhancement in the surface density between 1.5 and $2.0 \mathrm{kpc}$, and of course, there is a corresponding feature in the Newtonian rotation curves. But we see that there is also a feature at this position in the total rotation curve, even though there is a significant discrepancy between the Newtonian and detectable mass. The total rotation curve perfectly reflects details in the observed mass distribution even though the object is "dominated by dark matter" in the inner regions. 
This is an empirical point emphasized repeatedly by Sancisi [19]: For every feature in the surface brightness distribution (or gas surface density distribution) there is a corresponding feature in the observed rotation curve (and vice versa). I would add that with dark matter this seems rather unnatural. How is it that the dark matter distribution can match so perfectly the baryonic matter distribution? But with MOND, it is expected. What you see is all there is!

It has been argued that such features in rotation curves could be due to streaming motions in the gas associated with spiral arms or other such deviations from circular symmetry. Perhaps so, but then this would clearly be project for detailed modeling. Can nonaxially symmetric distortions create in detail the observed fluctuations in rotation curves in the presence of a dominant halo? This should be seen as an additional challenge for the dark matter paradigm.

In general, the success of MOND in accounting for galaxy rotation curves with only one free parameter, the $M / L$ of the visible disk which is usually found to have quite reasonable values, is striking. Whether the basic premise of MOND, that dynamics becomes nonNewtonian at low accelerations, is correct or not, the success of this simple algorithm implies that the detailed form of galaxy rotation curves is entirely determined by the distribution of visible matter. If the mass discrepancy is due to dark matter then this phenomenology demands explanation; it cannot be swept under the rug as being due to "messy astrophysics" or to unjustified claims that CDM halos possess an acceleration scale $[20,21]$. This goes well beyond the issue of whether or not dark halos have cusps. The point is that rotation curves indicate the presence of a cusp if there is a cusp in the light distribution; if rotation curves imply no cusp, then there is no cusp in the light distribution.

\section{Clusters of Galaxies: A Phenomenological Problem for MOND?}

It has been known for more than 70 years [22] that clusters of galaxies exhibit a significant discrepancy between the Newtonian dynamical mass and the observable mass, although the subsequent discovery of hot X-ray emitting gas goes some way in alleviating the original discrepancy. For an isothermal sphere of hot gas at temperature $\mathrm{T}$, the Newtonian dynamical mass within radius $r_{o}$, calculated from the equation of hydrostatic equilibrium, is

$$
M_{n}=\frac{r_{o}}{G} \frac{k T}{m}\left(\frac{d \ln (\rho)}{d \ln (r)}\right)
$$

where $m$ is the mean atomic mass and the logarithmic density gradient is evaluated at $r_{o}$. This dynamical mass turns out to be typically about a factor of five or six larger than the observed mass in hot gas and in the stellar content of the galaxies (see [23, Figure 7, left]).

With MOND, the dynamical mass (5) is given by

$$
M_{m}=\left(G a_{o}\right)^{-1}(k T m)^{2}\left(\frac{d \ln (\rho)}{d \ln (r)}\right)^{2},
$$

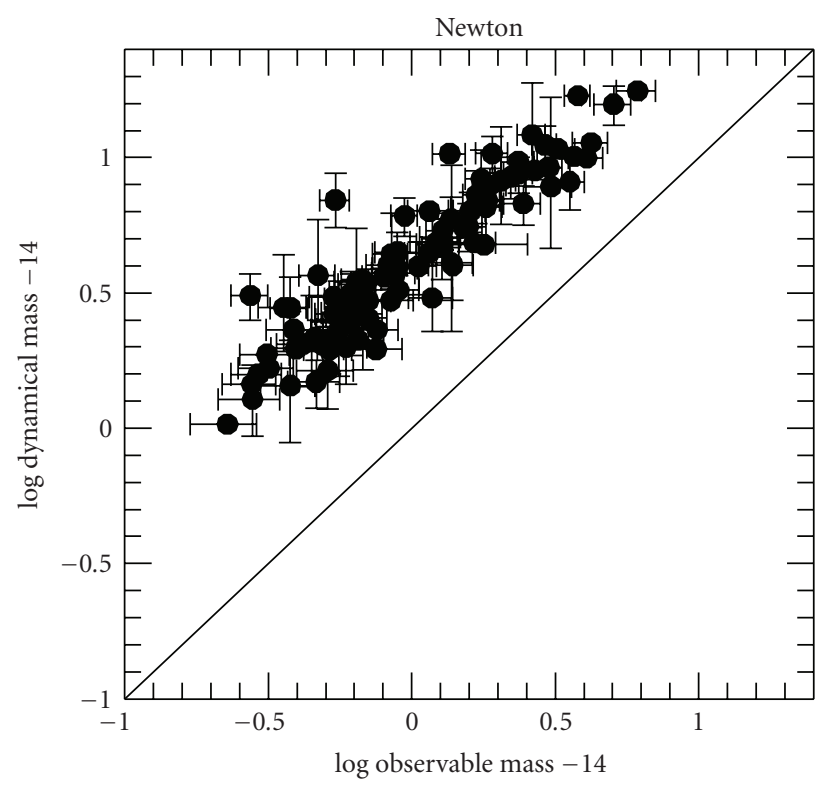

(a)

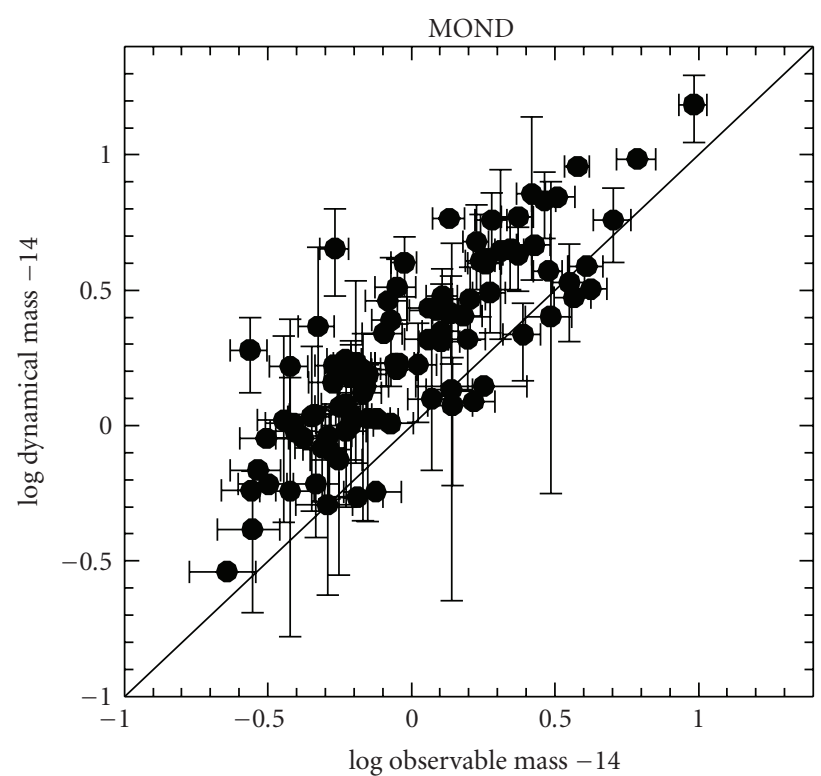

(b)

Figure 7: (a) The Newtonian dynamical mass of clusters of galaxies within an observed cutoff radius $\left(r_{\text {out }}\right)$ versus the total observable mass in 93 X-ray emitting clusters of galaxies. The solid line corresponds to $M_{\mathrm{dyn}}=M_{\mathrm{obs}}$ (no discrepancy). (b) The MOND dynamical mass within $r_{\text {out }}$ versus the total observable mass for the same X-ray emitting clusters [23].

and, using the same value of $a_{o}$ determined from nearby galaxy rotation curves turns out to be, on average, a factor of two or three larger than the observed mass (Figure 7(b)). The discrepancy is reduced but still present. This could be interpreted as a failure [24], or one could say that MOND predicts that the mass budget of clusters is not yet complete and that there is more mass to be detected [23]. The cluster missing mass could, for example, be in neutrinos of mass 
1.5 to $2 \mathrm{eV} \mathrm{[25],} \mathrm{or} \mathrm{in} \mathrm{"soft} \mathrm{bosons"} \mathrm{with} \mathrm{a} \mathrm{large} \mathrm{de} \mathrm{Broglie}$ wavelength [26], or simply in heretofore undetected baryonic matter. It would have certainly been a falsification of MOND had the predicted mass turned out to be typically less than the observed mass in hot gas and stars.

\section{Cosmology and Structure Growth}

MOND not only allows the form of rotation curves to be precisely predicted from the distribution of observable matter, but it also explains certain systematic aspects of the photometry and kinematics of galaxies and clusters. The presence of a preferred surface density in spiral galaxies and ellipticals-the so-called Freeman and Fish laws; the fact that pressure-supported nearly isothermal systems ranging from molecular clouds to clusters of galaxies are characterized by a specific internal acceleration $\left(a_{o}\right)$; the existence of a TF relation with small scatter-specifically a correlation between the baryonic mass and the asymptotically flat rotation velocity of the form $v^{4} \propto M$; the Faber-Jackson relation for ellipticals, and with more detailed modeling, the Fundamental Plane [27]; not only the magnitude of the discrepancy in clusters of galaxies but also the fact that massvelocity dispersion relation which applies to elliptical galaxies (7) extends to clusters (the mass-temperature relation). And it accomplishes all of this with a single new parameter with units of acceleration - a parameter determined from galaxy rotation curves which is within an order of magnitude of the cosmologically significant value of $\mathrm{cH}_{o}$. This is why several of us believe that, on an epistemological level, MOND is more successful than dark matter and, in fact, constitutes a falsification of dark matter on the scale of galaxies.

But, of course, MOND must fit into a larger picture. What are the larger-scale consequences of modified dynamics-specifically what are the implications for gravitational lensing and cosmology? Does MOND provide a mechanism for structure formation in a low density universe? These are questions which require a more basic theory underlying MOND, and there has been considerable progress in this respect as well.

I would first like to stress that MOND is not necessarily at odds with cherished physical principles. Soon after Milgrom's original papers, Felten [28] pointed out that the conservation of linear and angular momentum for an $\mathrm{N}$ body system is violated by Milgrom's simple formula. This had already been appreciated by Bekenstein and Milgrom [29] who reformulated MOND as a Lagrangian-based, modified Poisson equation.

$$
\nabla \cdot\left[\mu\left(\frac{|\nabla \phi|}{a_{o}}\right) \nabla \phi\right]=4 \pi G \rho
$$

where $\phi$ is the (scalar) gravitational field and the function $\mu(x)$ must have the asymptotic behavior required in the simple MOND prescription. In this form the usual conservation laws are respected. Moreover, this kind of nonlinear field equation appears in other contexts in physics; for example, it is identical to Maxwell's first equation in a nonlinear isotropic medium where the dielectric parameter is a function of the electric field strength.

Going beyond this nonrelativistic formulation, there has been considerable progress in constructing relativistic theories of gravity which, in the regime of very low field gradients, reproduce the phenomenology of MOND [2931]. This has culminated in the tensor-vector-scalar theory ( TeVeS) of Bekenstein, which is fully covariant and free of the anomalies of earlier attempts (such as superluminal propagation) [32]. The theory is complicated; it requires three additional parameters and a free function (one not specified by any a priori considerations but set to reproduce the phenomenology), but it does demonstrate that a covariant version of MOND is possible.

Milgrom [33] has considered the alternative point of view, that MOND, at a nonrelativistic level, may be due to a modification of the free particle action (modified inertia) resulting, possibly, from the interaction of a particle with vacuum fields [34]. Here, $a_{o}$ should appear as an effective constant for galactic systems, but, in general, the acceleration threshold for modified dynamics would be dependent upon the particle trajectory. In this case, one would expect Milgrom's original formula to be only an approximation to the truth for generalized particle motion.

Given there is not yet a generally accepted theoretical basis for MOND it might seem premature to consider cosmological implications. None the less, several general points can be made. First of all, it is clear that the experimental foundations of the standard Big Bang are so well established, that any underlying theory of MOND should not lead to a radically different cosmology, at least not in the early radiation-dominated Universe and certainly not at the epoch of nucleosynthesis. Then, to say that MOND is an alternative to dark matter does not imply that undetected or dark matter is nonexistent. In fact undetected baryonic matter must be present because $\Omega$ in visible matter is substantially less than $\Omega$ in baryons. Moreover, there are clear indications that at least some flavors of neutrinos have a nonvanishing mass [35], so there is a contribution of nonbaryonic dark matter to the total mass budget of the Universe-at least at a level comparable to the mean density of baryons in visible stars. But it would be contrary to the spirit of MOND if dark matter-baryonic or nonbaryonic — was a dominant constituent of galaxies; that is, MOND is inconsistent with the existence of cold dark matter (CDM) which clusters on small scales. So the question arises-how robust is the cosmological evidence for $\mathrm{CDM}$ at the level required for the now standard concordance model of the Universe $(\Lambda \mathrm{CDM})$ : roughly 70\% "dark" energy and 30\% CDM?

Support for the concordance model rests primarily upon the observations of distant supernovae [36], the angular power spectrum of the small-scale temperature fluctuations in the cosmic microwave background [37], and the amplitude of density fluctuations in the galaxy distribution as a function of scale. It is certainly true that the concordance model is consistent with these observations; however, this interpretation depends upon the validity of General Relativity and thereby, the validity of the Friedmann equation in describing the expansion history of the Universe. 
But if MOND is correct, then classical four-dimensional General Relativity does not give a proper description of reality on a cosmic scale. Indeed the peculiar composition of the Universe embodied by the concordance model has led some to suggest that modifications of standard General Relativity, and therefore late-time modifications of the Friedmann equation, are preferable. Often these modifications are based upon the currently popular braneworld scenarios [38]. But, recently, more ad hoc modifications have also been considered [39]. It is curious that such alternatives to dark energy are considered acceptable on aesthetic grounds, while a conceptually similar, but empirically justified, alternative to dark matter is not.

Of course, if we live in a Universe of only baryons and low-mass neutrinos, then how does structure form? After all a primary motivation for nonbaryonic dark matter is the necessity of forming the observed structure in the Universe by the present epoch via gravitational growth of very small density fluctuations. As we all know, nonbaryonic dark matter helps because it offers the possibility that fluctuations can begin growing before the epoch of hydrogen recombination. The expectation is that MOND, by providing stronger effective gravity in the limit of low accelerations, might also help, but in the absence of a proper theory, this question cannot be rigorously addressed (see, however, [40, 41]). Moreover, it appears that TeVeS can lead to structure formation on a level comparable to that of CDM [42].

Ideally, an underlying theory of MOND would make predictions on a scale other than extragalactic, and this would provide the possibility of a more definitive test. One general expectation is the appearance of deviations from $1 / r^{2}$ gravity in the outer solar system where the accelerations are becoming low. In this respect, the recently reported anomalous constant acceleration apparently detected beyond the orbit of Jupiter by the Pioneer spacecrafts [43] is most relevant. If confirmed, this would certainly indicate a breakdown of Newtonian dynamics in the low acceleration regime.

\section{Acknowledgment}

The author is very grateful to Moti Milgrom, Jacob Bekenstein, and Stacy McGaugh for useful discussions.

\section{References}

[1] M. Milgrom, "A modification of the Newtonian dynamics as a possible alternative to the hidden mass hypothesis," Astrophysical Journal, vol. 270, pp. 365-370, 1983.

[2] R. B. Tully and J. R. Fisher, "A new method of determining distances to galaxies," Astronomy \& Astrophysics, vol. 54, no. 3, pp. 661-673, 1977.

[3] W. J. G. de Blok and S. S. McGaugh, "Testing modified newtonian dynamics with low surface brightness galaxies: rotation curve fits," Astrophysical Journal, vol. 508, no. 1, pp. 132-140, 1998.

[4] S. S. Mcgaugh, J. M. Schombert, G. D. Bothun, and W. J. G. de Blok, "The baryonic tully-fisher relation," Astrophysical Journal, vol. 533, no. 2, part 2, pp. L99-L102, 2000.
[5] R. H. Sanders and M. A. W. Verheijen, "Rotation curves of Ursa major galaxies in the context of modified newtonian dynamics," Astrophysical Journal, vol. 503, no. 1, pp. 97-108, 1998.

[6] J. P. Ostriker and P. J. E. Peebles, "A numerical study of the stability of flattened galaxies: or, can cold galaxies survive?" Astrophysical Journal, vol. 186, pp. 467-480, 1973.

[7] K. C. Freeman, "On the disks of spiral and so galaxies," Astrophysical Journal, vol. 160, p. 811, 1970.

[8] R. J. Allen and F. H. Shu, "The extrapolated central surface brightness of galaxies," Astrophysical Journal, vol. 227, no. 2, pp. 67-72, 1979.

[9] A. H. Broeils,Ph.D. dissertation, University of Groningen, Groningen, The Netherlands, 1992.

[10] K. G. Begeman, "HI rotation curves of spiral galaxies," Astronomy \& Astrophysics, vol. 223, pp. 47-60, 1989.

[11] S. Casertano and J. H. van Gorkom, "Declining rotation curves: the end of a conspiracy?" Astronomical Journal, vol. 101, no. 4, pp. 1231-1241, 1991.

[12] K. G. Begeman, A. H. Broeils, and R. H. Sanders, "Extended rotationcurves of spiral galaxies: dark haloes and modified dynamics," Monthly Notices of the Royal Astronomical Society, vol. 249, pp. 523-537, 1991.

[13] M. Milgrom, "Isothermal spheres in the modified dynamics," Astrophysical Journal, vol. 287, pp. 571-576, 1984.

[14] S. M. Faber and R. E. Jackson, "Velocity dispersions and massto-light ratios for elliptical galaxies," Astrophysical Journal, vol. 204, pp. 668-683, 1976.

[15] R. H. Sanders and S. S. McGaugh, "Modified Newtonian dynamics as an alternative to dark matter," Annual Review of Astronomy and Astrophysics, vol. 40, pp. 263-317, 2002.

[16] W. J. G. de Blok, F. Walter, E. Brinks, C. Trachternach, S.-H. Oh, and R. C. Kennicutt, "High-resolution rotation curves and galaxy mass models from things," Astronomical Journal, vol. 136, no. 6, pp. 2648-2719, 2008.

[17] E. F. Bell and R. S. de Jong, "Stellar mass-to-light ratios and the Tully-Fisher relation," Astrophysical Journal, vol. 550, no. 1, pp. 212-229, 2001.

[18] R. A. Swaters, Ph.D. dissertation, University of Groningen, Groningen, The Netherlands, 1999.

[19] R. Sancisi, in IAU Symposium 222, D. J. Ryder, Ed.

[20] M. Kaplinghat and M. Turner, "How cold dark matter theory explains Milgrom's law," Astrophysical Journal, vol. 569, no. 1, pp. L19-L22, 2002.

[21] M. Milgrom, "Do modified Newtonian dynamics follow from the cold dark matter paradigm?" Astrophysical Journal, vol. 571, no. 2, pp. L81-L83, 2002.

[22] F. Zwicky, "Die Rotverschiebung von extragalaktischen Nebeln," Helvetica Physica Acta, vol. 6, pp. 110-127, 1933.

[23] R. H. Sanders, "The virial discrepancy in clusters of galaxies in the context of modified Newtonian dynamics," Astrophysical Journal, vol. 512, no. 1, pp. L23-L26, 1999.

[24] A. Aguirre, J. Schaye, and E. Quataert, "Problems for modified Newtonian dynamics in clusters and the Ly $\alpha$ forest?" Astrophysical Journal, vol. 561, no. 2, pp. 550-558, 2001.

[25] R. H. Sanders, "Clusters of galaxies with modified Newtonian dynamics," Monthly Notices of the Royal Astronomical Society, vol. 342, no. 3, pp. 901-908, 2003.

[26] R. H. Sanders, "A tensor-vector-scalar framework for modified dynamics and cosmic dark matter," Monthly Notices of the Royal Astronomical Society, vol. 363, no. 2, pp. 459-468, 2005.

[27] R. H. Sanders, "The fundamental plane of elliptical galaxies with modified Newtonian dynamics," Monthly Notices of the Royal Astronomical Society, vol. 313, no. 4, pp. 767-774, 2000. 
[28] J. E. Felten, “Milgrom's revision of Newton's laws: dynamical and cosmological consequences," Astrophysical Journal, vol. 286, pp. 3-6, 1984.

[29] J. D. Bekenstein and M. Milgrom, "Does the missing mass problem signal the breakdown of Newtonian gravity?" Astrophysical Journal, vol. 286, pp. 7-14, 1984.

[30] J. D. Bekenstein, in Proceedings of the 2nd Canadian Conference on General Relativity and Relativistic Astrophysics, A. Coley, C. Dyer, and T. Tupper, Eds., p. 487, World Scientific, Singapore, 1988.

[31] R. H. Sanders, "A stratified framework for scalar-tensor theories of modified dynamics," Astrophysical Journal, vol. 480, no. 2, pp. 492-502, 1997.

[32] J. D. Bekenstein, "Relativistic gravitation theory for the modified Newtonian dynamics paradigm," Physical Review D, vol. 70, no. 8, Article ID 083509, 28 pages, 2004.

[33] M. Milgrom, "Dynamics with a nonstandard inertiaacceleration relation: an alternative to dark matter in galactic systems," Annals of Physics, vol. 229, no. 2, pp. 384-415, 1994.

[34] M. Milgrom, "The modified dynamics as a vacuum effect," Physics Letters A, vol. 253, no. 5-6, pp. 273-279, 1999.

[35] M. C. Gonzalez-Garcia and Y. Nir, "Neutrino masses and mixing: evidence and implications," Reviews of Modern Physics, vol. 75, no. 2, pp. 345-402, 2003.

[36] S. Perlmutter, G. Aldering, G. Goldhaber, et al., "Measurements of $\Omega$ and $\Lambda$ from 42 high-redshift Supernovae," Astrophysical Journal, vol. 517, no. 2, pp. 565-586, 1999.

[37] D. N. Spergel, L. Verde, H. V. Peiris, et al., "First-year Wilkinson Microwave Anisotropy Probe (WMAP) observations: determination of cosmological parameters," Astrophysical Journal, vol. 148, no. 1, pp. 175-194, 2003.

[38] C. Deffayet, "Cosmology on a brane in Minkowski bulk," Physics Letters B, vol. 502, no. 1-4, pp. 199-208, 2001.

[39] S. M. Carroll, V. Duvvuri, M. Trodden, and M. S. Turner, "Is cosmic speed-up due to new gravitational physics?" http://arxiv.org/abs/astro-ph/0306438.

[40] R. H. Sanders, "The formation of cosmic structure with modified Newtonian dynamics," Astrophysical Journal, vol. 560, no. 1, pp. 1-6, 2001.

[41] A. Nusser, "Modified Newtonian dynamics of large-scale structure," Monthly Notices of the Royal Astronomical Society, vol. 331, no. 4, pp. 909-916, 2002.

[42] C. Skordis, D. F. Mota, P. G. Ferreira, and C. Boehm, "Large scale structure in bekenstein's theory of relativistic modified Newtonian dynamics," Physical Review Letters, vol. 96, no. 1, Article ID 011301, 4 pages, 2006.

[43] J. D. Anderson, P. A. Laing, E. L. Lau, A. S. Liu, M. M. Nieto, and S. G. Turyshev, "Indication, from pioneer 10/11, Galileo, and Ulysses data, of an apparent anomalous, weak, long-range acceleration," Physical Review Letters, vol. 81, no. 14, pp. 2858 2861, 1998. 

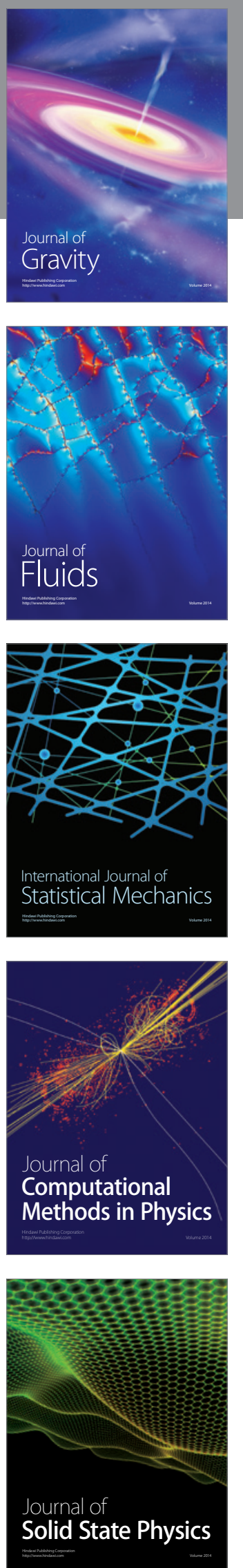

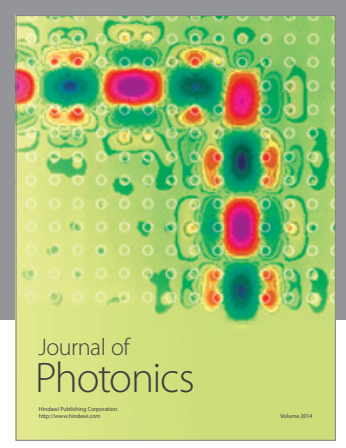

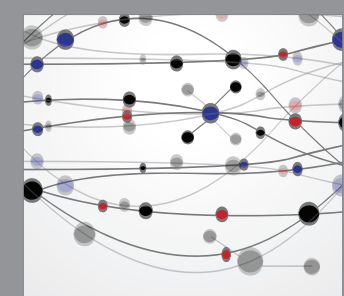

The Scientific World Journal
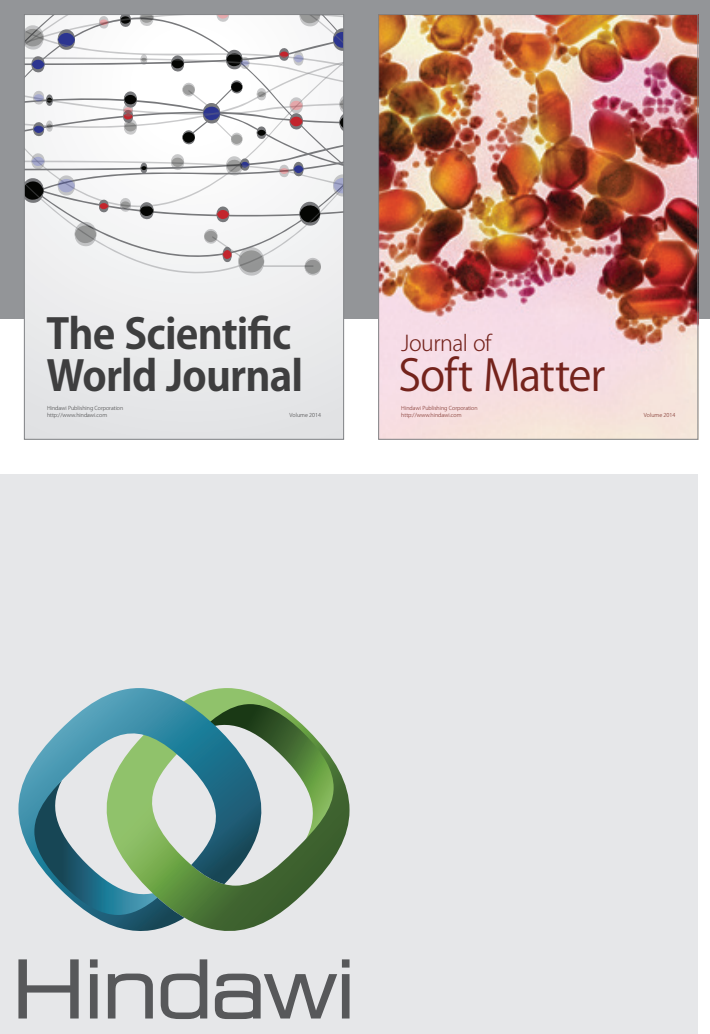

Submit your manuscripts at

http://www.hindawi.com
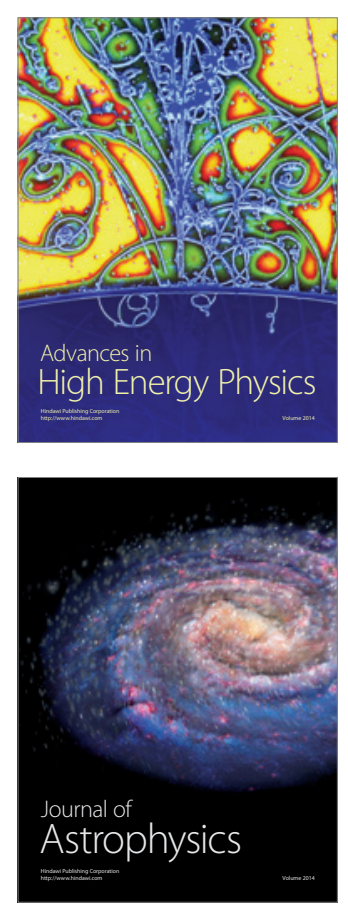
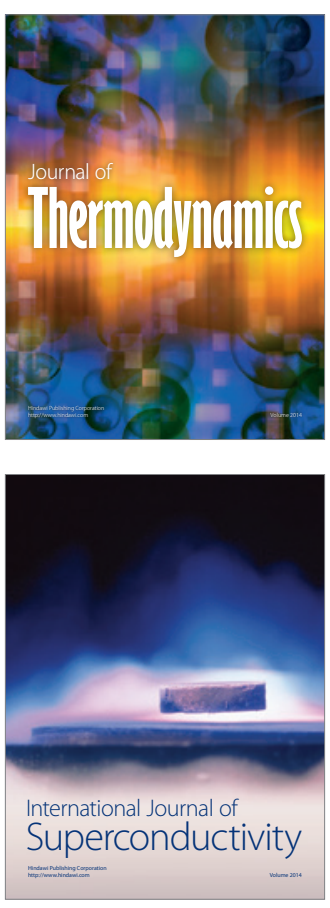
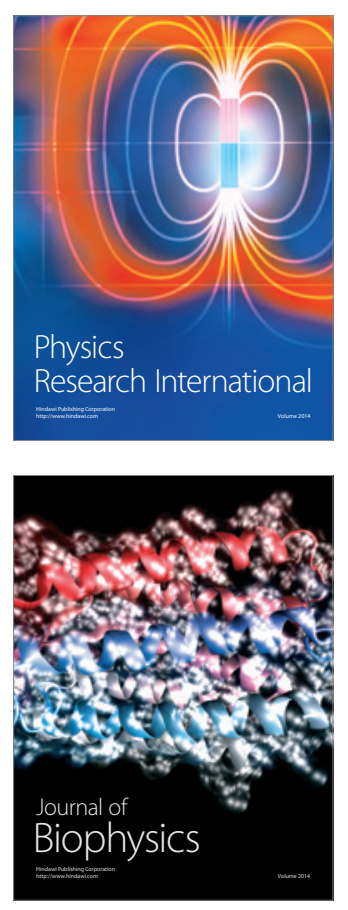
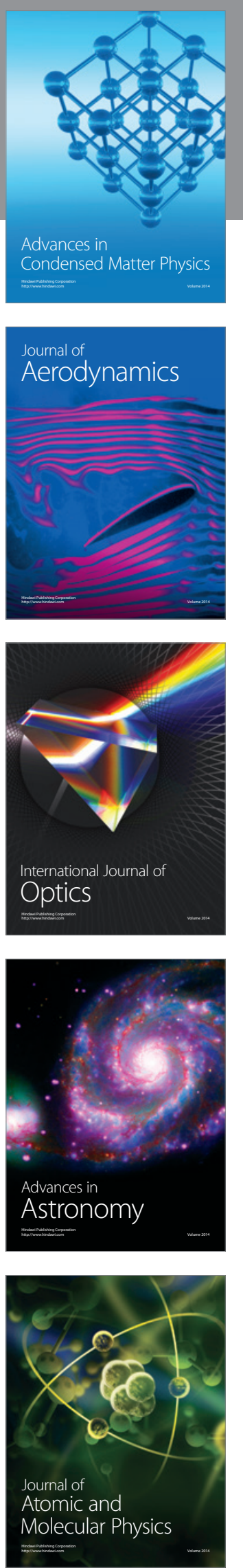